\title{
1 Role of gene body methylation in coral acclimatization and adaptation.
}

3 Groves Dixon ${ }^{1}$, Yi Liao ${ }^{1}$, Line K. Bay ${ }^{2}$ and Mikhail V. Matz ${ }^{1}$

4

$5{ }^{1}$ Department of Integrative Biology, University of Texas at Austin, 2401 Speedway Ave, Austin,

6 TX 78712, USA

$7 \quad{ }^{2}$ Australian Institute of Marine Science, PMB 3, Townsville, Queensland 4810, Australia

10 Corresponding Author:

$11 \quad$ Mikhail Matz

12 matz@utexas.edu

$512-992-8086$

17 Genome-wide shifts in gene body methylation predict gene expression and fitness during

18 acclimatization but do not contribute to epigenetic divergence between populations. 


\section{Abstract}

22 Gene body methylation (GBM) has been hypothesized to modulate responses to environmental

23 change, including transgenerational plasticity, but the evidence thus far has been lacking. Here

24 we show that coral fragments reciprocally transplanted between two distant reefs respond with

25 genome-wide increase or decrease in GBM disparity among genes. Surprisingly, this simple

26 genome-wide adjustment predicted broad-scale gene expression changes and fragments' fitness

27 in the new environment. This supports GBM's role in acclimatization, which may consist in

28 modulating the expression balance between environmentally-responsive and housekeeping

29 genes. At the same time, constitutive differences in GBM between populations did not align

30 with plastic GBM changes upon transplantation and were mostly observed among $F_{\mathrm{ST}}$ outliers,

31 indicating that they arose through genetic divergence rather than through transgenerational

32 inheritance of acquired GBM states.

GBM is a taxonomically widespread epigenetic modification the function of which

35 remains unclear $(1,2)$. GBM is bimodally distributed among genes: it is high in ubiquitously

36 expressed housekeeping genes and low in inducible genes $(2,3)$. Only the detrimental effect of

37 GBM is well understood: GBM causes hypermutability in protein-coding regions (4). Indeed, in

38 humans, GBM is the primary driver of deleterious parent-age-related mutations (5). To merit

39 pervasive evolutionary conservation, the fundamental biological function of GBM must be

40 important enough to outweigh this risk (1). Among putative cellular functions of GBM, 
41 suppression of intragenic transcription initiation is currently the most studied $(6,7)$ but still

42 remains controversial (8). The ecological genomic literature has long expected that GBM (as well

43 as other epigenetic marks) respond to the environment and assist acclimatization by modulating

44 gene expression, possibly across generations (9-11). Still, thus far the only well-documented

45 case of GBM responding to the environment and resulting in phenotypic change is caste

46 determination in social insects depending on diet (12). Beyond this highly taxon-specific

47 example, GBM response to the environment has been reported in a recent paper on coral

48 acclimatization to acidic conditions (13); notably, GBM change did not specifically affect genes

49 expected to be involved in such acclimatization. One way to address the role of GBM in

50 adaptation would be to examine differences between populations, as has been recently done in

51 plants $(14,15)$; however, this interpretation requires disentangling the effect of environment

52 from the effect of genetic divergence among populations (16). As for transgenerational

53 plasticity, for GBM to be involved in this process it must be both responsive to the environment

54 and heritable. This somewhat contradictory combination of properties has not yet been

55 demonstrated for GBM in any study system.

56 Here, we used a reciprocal transplantation framework (17) to test for the roles of GBM,

57 gene expression, and genetics in local adaptation and acclimatization in a reef-building coral

58 Acropora millepora. In corals, the possibility of clonal replication makes disentangling the

59 effects of genotype and environment very straightforward, solving a major problem of

60 ecological epigenetics (16). We compared corals from two reefs, Orpheus Island in the central

61 sector of the Great Barrier Reef (GBR) and Keppel Island in the south of GBR (Fig. 1 A). These 
62 reefs are notably different in temperature (Fig. 1 B) as well as several other abiotic parameters

$63(18)$ and host slightly genetically divergent $A$. millepora populations $(19,20)$. Fifteen coral

64 colonies from each reef were halved and reciprocally transplanted between the sites for three

65 winter months (Fig. 1 A, B). In this way, each individual genotype was simultaneously exposed to

66 two distinct reef conditions. After three months in the field, tissue samples were collected from

67 each fragment and assayed for genome-wide gene expression using TagSeq (21) and for DNA

68 methylation using MBD-seq (22). These data were analyzed in the context of several fitness

69 proxies and genetic distances between individuals (based on genetic polymorphisms detected in

70 the MBD-seq data). All experimental corals were dominated by Symbiodinium clade C, which

71 was established by analyzing Symbiodinium-matching MBD-seq reads.

For both Keppel- and Orpheus-origin corals fitness proxies were higher at Keppel,

73 possibly due to higher concentration of inorganic nutrients there $(18,19)$. For Keppel-origin

74 corals the difference in performance was predominantly in weight gain (i.e., skeletal growth)

75 while for Orpheus-origin corals the difference was mostly in internal stores (lipids, proteins and

76 carbohydrates, Fig. 1 C). This indicates that (i) Keppel and Orpheus environments are indeed

77 different from corals' perspective, (ii) both populations are capable of plastic responses to these

78 differences, and (iii) there is also constitutive divergence in physiology between populations. 

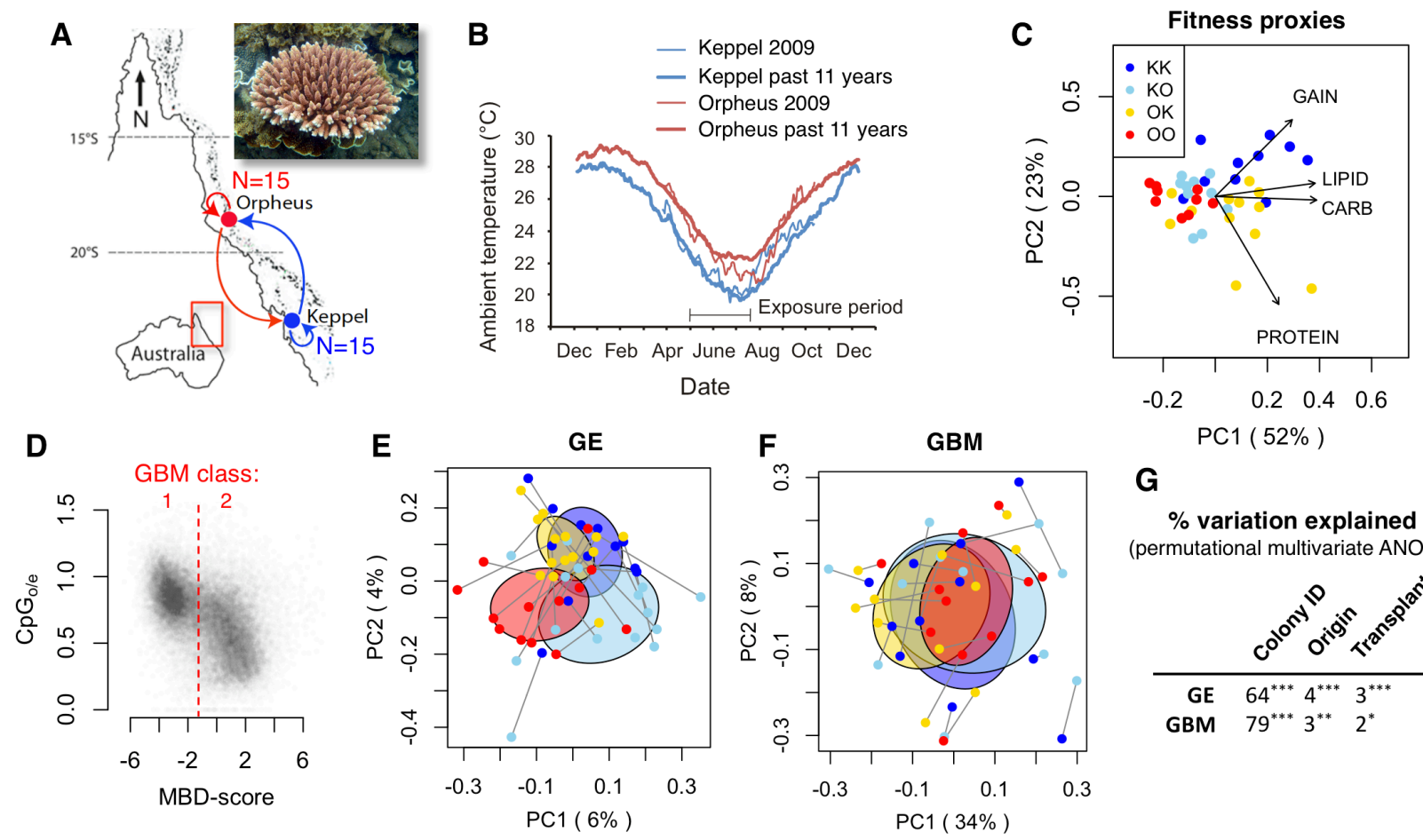

G

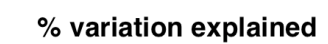
(permutational multivariate ANOVA)

82 Figure 1. Overview of the experiment. A: Design of reciprocal transplantation. Inset - Acropora millepora.

83 B: Temperature profiles at the transplantation sites, historical (thick lines) and during the year of

84 experiment (thin lines). C: Principal component analysis of the four fitness proxies: weight gain, lipid,

85 protein and carbohydrate content. In the sample names, the first letter is the origin location and the

86 second letter is transplantation location. D: Scatter plot of the measure of depletion of CpG dinucleotides

87 (ratio of observed vs. expected number of $\mathrm{CpGs}, \mathrm{CpG}_{\mathrm{O} / \mathrm{E}}$ ) against $\mathrm{MBD}$-score ( $\log _{2}$ of fold-difference in

88 read counts between MBD-captured and flow-through fraction) for each gene. The MBD-score threshold

89 defining the two GBM classes is indicated. E and F: Principal coordinate analysis of gene expression (E)

90 and gene body methylation (F). Lines connect fragments of the same original colony; the color scheme

91 follows the legend on panel C. For GE, PC1 corresponds to the colony's origin and PC2 - to the

92 transplantation site; no such partitioning is visible for GBM. G: Percentage of variation in GE and GBM 
93 explained by colony identity (i.e., by similarity among clonal fragments, or broad-sense heritability),

94 colony's origin and transplantation site. ${ }^{* * *} p<0.001 ;{ }^{* *} p<0.01 ;{ }^{*} p<0.05$.

To determine the characteristic level of GBM for each gene we used MBD-seq results for

9612 samples (three per each experimental group, KK, KO, OK, and OO) for which both MBD-

97 captured and flow-through fraction were sequenced. Logarithm with the base 2 of each gene's

98 abundance in captured vs. flow-through fractions ("MBD-score" (22)) exhibited the expected

99 bimodal distribution (Fig. S1 A) and showed strong correlation (Fig. 1 D) with the established

100 proxy of historical methylation level, the ratio of observed to expected numbers of CpG

101 dinucleotides ("CPG/E" (3)). In addition to this genome-wide validation of our methylation data,

102 we validated GBM results by bisulfite-sequencing 13 amplicons representing different MBD-

103 score classes (Fig. S1 B, C). Genes were assigned to either low-methylated class (class 1) or high-

104 methylated class (class 2) based on the MBD-score threshold indicated on Fig. 1 D. Relative

105 quantification results based only on MBD-captured read data were nearly identical to those

106 obtained when using both captured and flow-through fractions (Fig. S2). For the remaining 32

107 samples, we sequenced only the MBD-captured fraction.

Multivariate analysis of gene expression (GE, Fig. $1 \mathrm{E}$ ) and GBM (Fig. $1 \mathrm{~F}$ ) revealed that

109 GBM is more consistent across fragments of the same original colony than GE, resulting in the

110 estimate of broad-sense heritability of 0.79 compared to 0.64 for GE (Fig. 1G, Fig. S3 and S4). In

111 addition, significant effects of origin and transplantation site were observed for GE and GBM,

112 both more pronounced for GE (Fig. $1 \mathrm{E}$ and G). 
114 consisted mainly in genome-wide reduction of disparity between high- and low-methylated

115 genes: highly-methylated genes became less methylated and low methylated became more

116 methylated (Fig. 2 A). This change was mirrored by less pronounced but clearly reciprocal GBM

117 adjustment in Keppel corals transplanted to Orpheus (Fig. 2 B, D) and recapitulated the

118 difference between fragments planted in their native environment (Fig. 2 C). Both exons and

119 introns underwent this change (Fig. S5). Absolute changes in methylation included both positive

120 and negative shifts (Fig. S6 A, B), indicating that the change in relative GBM levels among genes

121 is not due to biased genome-wide methylation increase or decrease. Methylation levels of

122 intergenic regions and repeated elements remained relatively constant compared to genic

123 regions (Fig. S6 C, D).

125 much broader range of fold-changes compared to GBM response (Fig. 2 D). Remarkably, gene

126 expression changes correlated with low-and high-methylated gene classes and were in the

127 opposite direction relative to GBM changes (Fig. 2 F, G). Although at the gene level the

128 correlation between GE and GBM was weak (but still highly significant, Fig. S7), it was very

129 strong when comparing broader functional groups of genes (Gene Ontology categories, Fig. $2 \mathrm{H}$

130 and Fig. S8).

131 To characterize GBM disparity among genes in each sample, we computed GBM class

132 difference ("GBMcd") as difference in mean $\log _{2}(G B M)$ between high- and low-methylated gene 
133 classes (Fig. 2 I). This measure aligned nearly perfectly with the first principal component of

134 GBM variation for the whole experiment, explaining 33\% of total variation (Fig. $1 \mathrm{~F}$ and Fig. $2 \mathrm{~J}$,

135 which show the same PCA ordination but are colored differently). For comparison, the next

136 principal component explains only $8 \%$ of GBM variation.
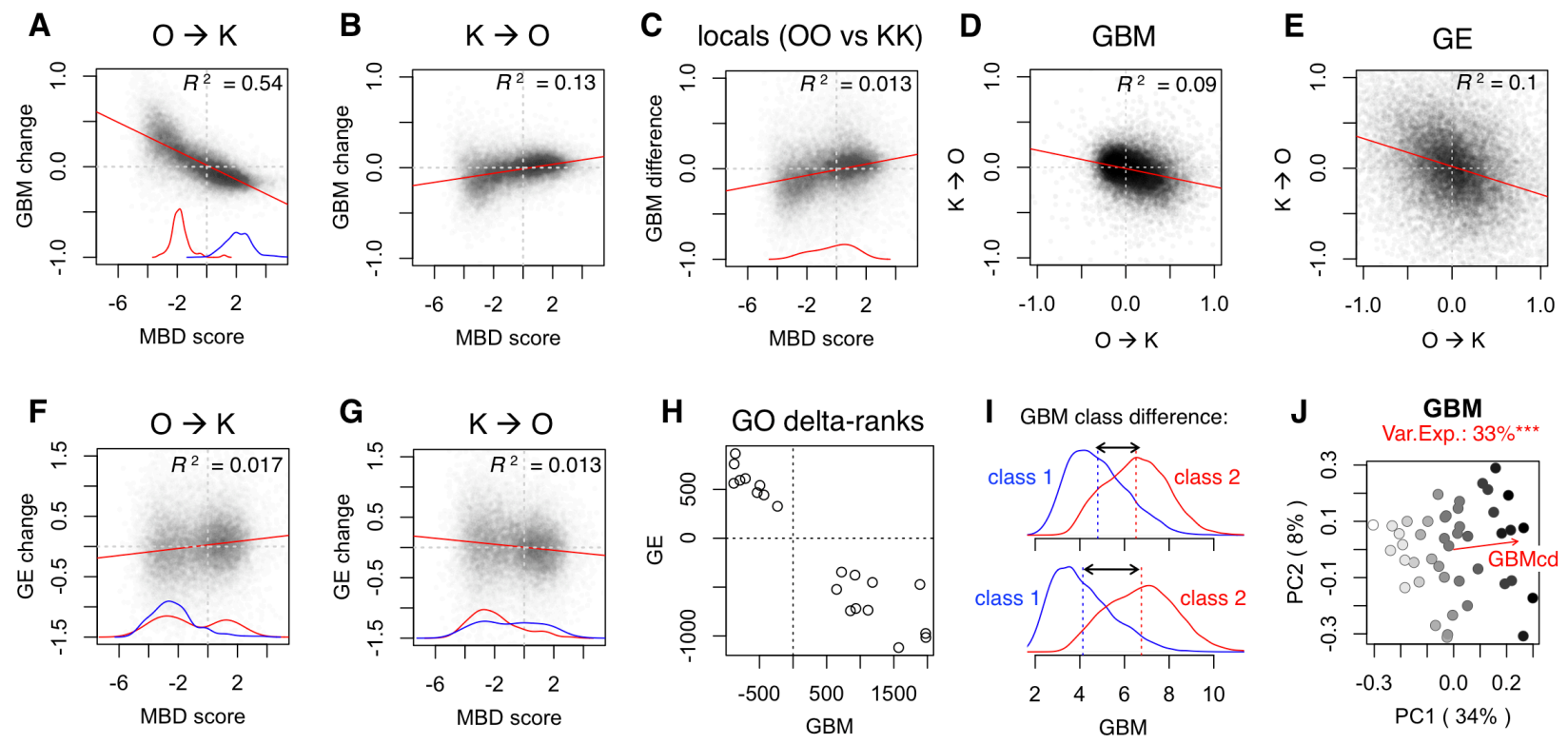

138 Figure 2. Changes in gene body methylation (GBM) and gene expression (GE) in response to

139 transplantation ("away" relative to "home"). On all panels, GE and GBM measures are $\log _{2}$ fold-changes.

140 A though C: Differences in GBM in fragments transplanted from Orpheus to Keppel (A), from Keppel to

141 Orpheus (B), and fragments planted in their home environment (C) plotted against MBD-score for each

142 gene. Red and blue curves near the x-axis are density plots of significantly up-and down-regulated genes

143 (there were none for the $\mathrm{K} \rightarrow \mathrm{O}$ experiment). $\mathrm{D}$ and $\mathrm{E}$ : Correlation between GBM (D) and GE (E) changes in

144 reciprocally transplanted fragments. F and G: Change in GE in fragments transplanted from Orpheus to

145 Keppel (F) and from Keppel to Orpheus (G) plotted against MBD-score for each gene (compare to panels

146 A and B). H: Plot of delta-ranks for 20 Gene Ontology categories that were significantly enriched with 
147 either up-regulated genes (positive delta-rank) or down-regulated genes (negative delta-rank) in both GE

148 and GBM analyses. I: Calculation of GBM class difference (GBMcd), a proxy of genome-wide GBM

149 disparity among genes. Each panel shows two density plots of GBM levels for the two GBM gene classes

150 (see Fig. 1 D), with the mean for each class indicated by a dashed line. The top panel shows the sample

151 with the lowest GBMcd, bottom panel - the sample with the highest GBMcd. J: Principal coordinate

152 biplot (the same ordination as on Fig. $1 \mathrm{~F}$ ) colored according to GBMcd. The purple vector shows the

153 direction of correlation of GBMcd with the ordination. All correlations with reported $\mathrm{R}^{2}$ are significant at

154 the $p<0.0001$ level.

Our data do not support transgenerational inheritance of acquired GBM changes. If

156 plastic changes in GBM were transmitted across generations, one would expect constitutive

157 GBM differences between Orpheus- and Keppel-origin corals to parallel the GBM response

158 induced upon transplantation. Instead, constitutive differences between Orpheus and Keppel

159 (differences between all Orpheus-origin and all Keppel-origin fragments, irrespective of

160 transplantation site) were in the opposite direction compared to plastic changes (compare Fig. 3

161 A and Fig. 2 C), did not predominantly affect genes at the opposite ends of MBD-score spectrum

162 (compare density curves on Fig. $2 \mathrm{~A}$ and Fig. $3 \mathrm{~A}$ ), and were positively rather than negatively

163 associated with differences in GE (Fig. 3 B). In addition, genes showing significant constitutive

164 differences in GBM also showed elevated $F_{\text {ST }}$ (Fig. 3 C) unlike genes undergoing significant plastic

165 changes (Fig. S9). This indicates that the mechanism giving rise to constitutive GBM changes

166 between populations is unrelated to the mechanism of plasticity and is most likely genetic

167 divergence. 

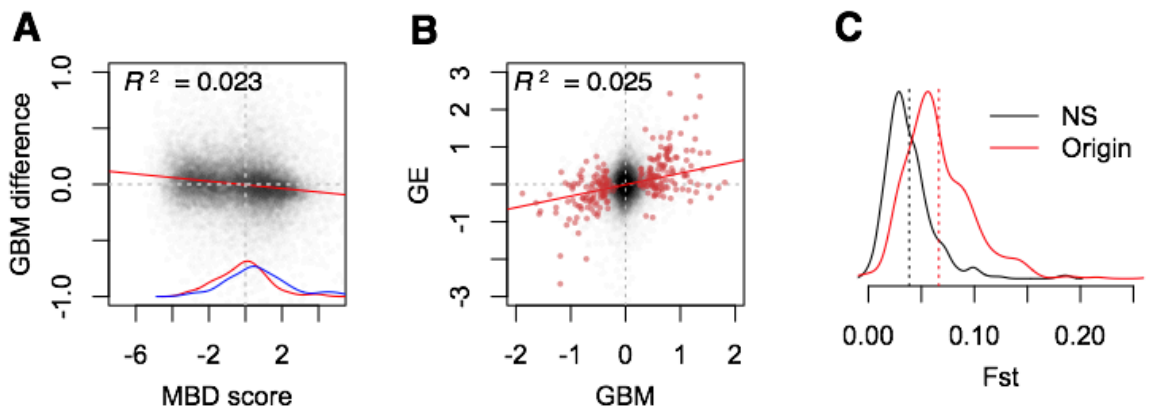

169 Figure 3. Constitutive $\log _{2}$ fold-differences in gene body methylation (GBM) and gene expression (GE)

170 between populations (Orpheus relative to Keppel). A: Difference in GBM plotted against MBD-score for

171 each gene. Red and blue curves by the x-axis are density plots of significantly up- and down-regulated

172 genes. B: Correlation between GBM and GE differences. Red points are genes significantly different in

173 GBM at 10\% FDR level. This correlation is positive, while correlations between plastic GBM and GE

174 changes are negative (Fig. $2 \mathrm{H}$ and Fig. S7). E: Density plot of between-population $F_{\mathrm{ST}}$ for genes showing

175 significant constitutive difference in GBM (red line) compared to 500 randomly chosen non-significant

176 genes (black line). Dashed vertical lines mark the mean of each dataset. All correlations with reported $\mathrm{R}^{2}$

177 are significant at $p<0.0001$ level.

To see whether plastic GBM changes were related to acclimatization (rather than, for

179 example, overall stress in response to transplantation) we used Differential Analysis of Principal

180 Components (DAPC, Fig. 4 A-C) to test whether GBM similarity to the local population (Fig. 4 D)

181 predicted the transplanted fragment's fitness in the new environment. The resulting "GBM

182 similarity to locals" was highly correlated with the first principal component of measured fitness

183 proxies, the most strongly with the weight gain (Fig. 4 E). This correlation held both before and

184 after (as shown on Fig 4 E) controlling for overall higher fitness of Orpheus corals transplanted 
185 to Keppel. This result indicates that a coral's GBM profile, despite being highly variable among

186 individuals (Fig $1 \mathrm{~F}, \mathrm{G}$ ), also reflects adaptation and acclimatization to local environmental

187 conditions. Interestingly, the same analysis performed for GE and genotypes (GT) revealed no

188 relationships with fitness (Fig. 4 E). One explanation of this could be the difference in time scales

189 on which the effects of GE and GT on fitness would be detectable: compared to the three-

190 month length of our experiment, GE might be linked to fitness on shorter time scales, and GT -

191 on longer time scales.
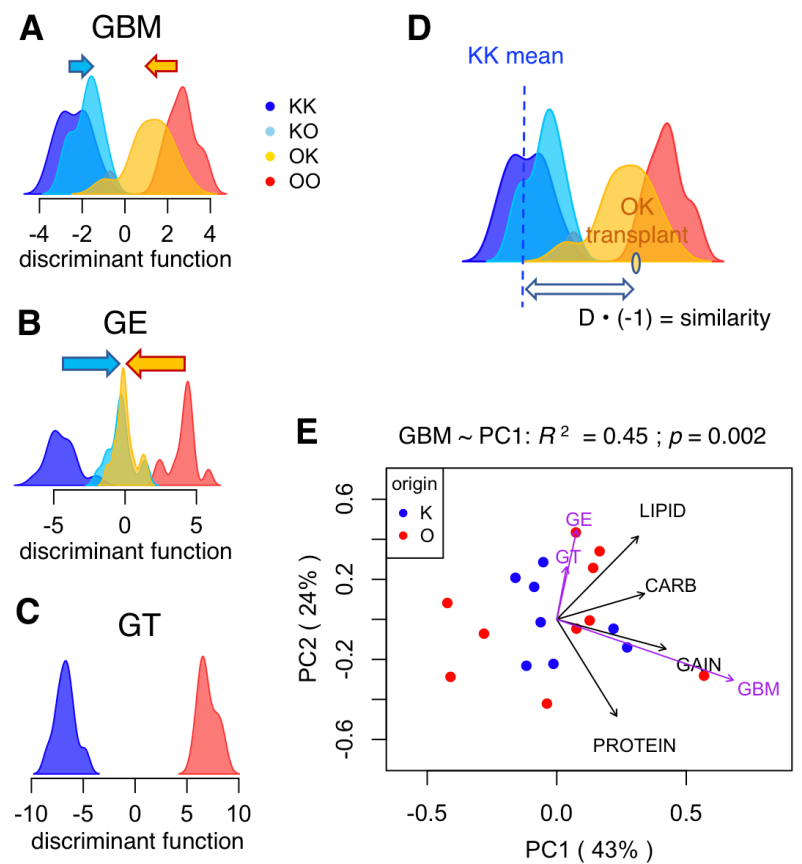

193 Figure 4. Relationship between gene body methylation (GBM), gene expression (GE), and genotype (GT)

194 with fitness proxies in corals transplanted into novel environment. A-C: Density plots of discriminant

195 function values for each variable. The functions were developed to distinguish between coral fragments

196 in their native environment (KK vs. OO) and applied to transplanted fragments (KO and OK) to quantify

197 their adaptive plasticity (ability to shift towards local values). Arrows indicate mean plasticity. D: 
199 fitness proxies in fragments transplanted to another reef, corrected for the overall higher fitness of

200 Orpheus corals transplanted to Keppel. Fit of "similarity to locals" for GBM, GE and GT onto this

201 ordination is shown as purple vectors scaled by correlation coefficient. Only GBM fit is statistically

202 significant.

204 prompts the question: which one is likely to be the leading cause? The magnitude of GBM

205 changes is much lower than changes in GE (Fig. 2 D, E), which suggests that GE might be the

206 primary driver. However, considering the simplicity of plastic GBM changes - genome-wide

207 exaggeration or mitigation of GBM disparity among genes - it is more parsimonious to assume

208 that this is the leading change driven by the environment, which then translates into broad-

209 scale functional shifts in gene expression (Fig. $2 \mathrm{H}$ and Fig. S8). In this way, genome-wide

210 adjustment of gene activity could be achieved by modulating a single process that governs the

211 disparity in GBM among genes.

213 broad gene classes: the low-methylated environmentally-responsive genes and highly-

214 methylated housekeeping genes. In the higher-quality environment of the Keppel island, where

215 corals attain higher fitness (Fig. 1C), GBM disparity change is associated with suppression of the

216 environmentally responsive genes and up-regulation of housekeeping genes (Fig. $2 \mathrm{~F}$ and Fig.

217 S8). Conversely, at the low-quality Orpheus Island the balance shifts the other way, from

218 housekeeping to environmental responsiveness (Fig. $2 \mathrm{G}$ and Fig. S8). We propose that mediating 
such coarse adjustment of physiology in response to the environment is the ecological function

220 of GBM.

221

References (Main paper)

223

224

1. D. Zilberman, An evolutionary case for functional gene body methylation in plants and

225

226 animals. Genome Biol. 18, 87 (2017).

2. A. J. Bewick, R. J. Schmitz, Gene body DNA methylation in plants. Curr. Opin. Plant Biol. 36, 103-110 (2017). Methylation. Mol Biol Evol. 29, 1907-1916 (2012).

4. A. P. Bird, DNA methylation and the frequency of $C p G$ in animal DNA. Nucleic Acids Res. 8, 1499-1504 (1980).

5. L. C. Francioli et al., Genome-wide patterns and properties of de novo mutations in humans. Nat. Genet. 47, 822-826 (2015).

6. A. K. Maunakea et al., Conserved role of intragenic DNA methylation in regulating alternative promoters. Nature. 466, 253-7 (2010).

7. F. Neri et al., Intragenic DNA methylation prevents spurious transcription initiation. Nature. 543, 72-77 (2017).

8. D. Jjingo et al., On the presence and role of human gene-body DNA methylation.

240 9. S. B. Roberts, M. R. Gavery, Is there a relationship between DNA methylation and phenotypic plasticity in invertebrates? Front. Physio. 2, 1-5 (2012).

242 10. G. E. Hofmann, Ecological Epigenetics in Marine Metazoans. Front. Mar. Sci. 4, 4 (2017).

243 11. R. Feil, M. F. Fraga, Epigenetics and the environment: emerging patterns and implications. Nat. Rev. Genet. 13, 97 (2012).

245 12. H. Yan et al., DNA Methylation in Social Insects: How Epigenetics Can Control Behavior and Longevity. Annu. Rev. Entomol. 60, 435-452 (2015). 
13. Y. J. Liew et al., Epigenome-associated phenotypic acclimatization to ocean acidification in a reef-building coral. Sci. Adv. 4, eaar8028 (2018).

14. P. F. Gugger, S. Fitz-Gibbon, M. Pellegrini, V. L. Sork, Species-wide patterns of DNA methylation variation in Quercus lobata and their association with climate gradients. Mol. Ecol. 25, 1665-1680 (2016).

15. T. E. Keller, J. R. Lasky, S. V. Yi, The multivariate association between genomewide DNA methylation and climate across the range of Arabidopsis thaliana. Mol. Ecol. 25, 18231837 (2016).

16. J. Hu, R. D. H. Barrett, Epigenetics in natural animal populations. J. Evol. Biol. (2017), doi:10.1111/jeb.13130.

17. T. J. Kawecki, D. Ebert, Conceptual issues in local adaptation. Ecol. Lett. 7, 1225-1241 (2004).

18. C. Lønborg et al., "Marine Monitoring Program: Annual report for inshore water quality monitoring 2014 to 2015" (Australian Institute of Marine Science and JCU TropWATER, Townsville, 2016), (available at http://elibrary.gbrmpa.gov.au/jspui/handle/11017/3050).

19. M. V. Matz, E. A. Treml, G. V. Aglyamova, L. K. Bay, Potential and limits for rapid genetic adaptation to warming in a Great Barrier Reef coral. PLOS Genet. 14, e1007220 (2018).

20. M. J. H. Van Oppen, L. M. Peplow, S. Kininmonth, R. Berkelmans, Historical and

21. E. Meyer, G. V. Aglyamova, M. V. Matz, Profiling gene expression responses of coral larvae (Acropora millepora) to elevated temperature and settlement inducers using a novel RNA-Seq procedure. Mol. Ecol. 20 (2011), doi:10.1111/j.1365-294X.2011.05205.x. Basal Metazoan. Mol. Biol. Evol. 33, 2285-2293 (2016). 


\section{Materials and Methods}

276

277

278

279

280

281

282

283

284

285

286

287

288

289

290

291

292

293

294

Sample sizes

The experiment started with 15 colonies from each of the two sites, which were halved

and transplanted, resulting in a total of 60 fragments distributed across four study groups 15 samples each: two "natives" (KK and OO), and two "transplants" (OK and KO, Fig. 1C). Although

not all original samples were successfully analyzed using all approaches (Table S1), in the end each of these four groups included 11 fragments representing unique coral genotypes and paired by genotype between "home" and "away" groups that were analyzed for GBM, gene expression, genotype, and fitness proxies.

Reciprocal Transplantation Experiment

Field work was conducted with permission from the Great Barrier Reef Marine Park

Authority (Research permit G09/29894.1) as described previously (23). Reciprocal

transplantations were undertaken between two environmentally distinct study sites (Miall Island in the Keppel Island group: $23^{\circ} 09 \mathrm{~S} 150^{\circ} 54 \mathrm{E}$ and Hazard Bay on Orpheus Island $18^{\circ} 37 \mathrm{~S}$ $146^{\circ} 29 \mathrm{E}$ ) separated by 4.5 degrees of latitude on the Great Barrier Reef (Figure $\left.1 \mathrm{~A}\right)$. On the 23rd of April (Orpheus) and 4th of May (Keppels) 2010 fifteen colonies were collected from wild populations from each site and split in two. One half of each colony was replaced in its native habitat, while the second half was transplanted to the alternate study site. Samples from all coral fragments were collected at midday after three months (9th July 2010 at Orpheus, 
295 14th July at Keppels) frozen in liquid nitrogen, then transferred into RNAlater (Ambion, Austin,

296 TX, USA) for gene expression and DNA methylation profiling.

Total colony skeletal growth was evaluated using wet buoyant weight as in Jokiel,

300 Maragos, \& Franzisket (1978). Measurements from beginning and end of the experiment were

301 expressed as percent daily weight gain.

The energetic condition of corals was determined by the analysis of total protein,

305 carbohydrate and lipid content. Coral tissue was removed from an average of $8.6 \mathrm{~cm}^{2}$ of coral

306 branch (mean $\pm \mathrm{SE}=0.18$ ) using an air gun in $12-15 \mathrm{ml}$ of $0.2 \mu \mathrm{M}$ filtered sea-water $(\mathrm{FSW})$. The

307 coral slurry was homogenized and centrifuged at $1500 \mathrm{~g}$ for $5 \mathrm{~min}$ at $4^{\circ} \mathrm{C}$ to separate coral and

308 endosymbiotic algal components. The coral slurry was aliquoted for protein, carbohydrate and

309 lipid assays and frozen at $-20^{\circ} \mathrm{C}(10 \mathrm{ml}$ for lipid, $0.5 \mathrm{ml}$ for protein and carbohydrate). The algal

310 component was re-suspended in $2.75 \mathrm{ml}$ of $0.2 \mu \mathrm{M}$ FSW. Coral tissue surface area was

311 determined from cleaned (10\% bleach for $24 \mathrm{hrs}$ ) skeletons of water-blasted samples using a

312 twice dip paraffin wax method (25). Surface area of coral branches was determined by the

313 weight gain of the second wax layer from a session specific standard curve $\left(R^{2}\right.$ were $\left.0.97-0.98\right)$

314 using seven nylon cylinders of known surface area $\left(5-48 \mathrm{~cm}^{2}\right)$. 
Protein analysis

318 incubated at $90^{\circ} \mathrm{C}$ for one hour and spun at $3000 \mathrm{~g}$ for 5 minutes to separate cell-debris from

319 the solution and $500 \mu \mathrm{L}$ clear supernatant was transferred onto a 96-well format. Protein

320 concentration was quantified in three technical replicates of $50 \mu$ of coral protein extract using

321 a microplate Peterson - Lowry assay following the manufacturer's recommendations (Sigma:

322 TP0300). Blank $0.5 \mathrm{M} \mathrm{NaOH}$ samples and BSA protein standards (50, 100, 200, 300 and $400 \mu \mathrm{g} /$

$323 \mathrm{ml}$ ) were run in duplicates on each plate and absorbance was read at $595 \mathrm{~nm}$ on a

324 spectrophotometer. Standard curves had $R^{2}$ of $0.97-0.99$. The coefficient of variation (CV) was

325 calculated among technical replicates and deemed acceptable when $<10 \%$. For samples with a

326 CV > 10\%, single deviant outliers (>1 SD deviant from other two measurements) were identified

327 and omitted. Total protein content per sample was expressed as average content (mg) of the

328 technical replicates per $\mathrm{cm}^{2}$ of coral branch surface area.

Total carbohydrate content estimates were obtained from the average of three technical

332 replicates of $50 \mu \mathrm{L}$ coral slurry using D-glucose as a standard (26). Blank water samples and BSA

333 protein standards $(20,50,100,200,500$ and $1000 \mu \mathrm{g} / \mathrm{ml})$ were run in triplicate on each plate

334 and absorbance was read at $485 \mathrm{~nm}$ on a spectrophotometer. Standard curves had $\mathrm{R}^{2}$ of $0.96-$

3350.99 and total carbohydrate content per sample was expressed in average $\mathrm{mg}$ of the technical

336 replicates per $\mathrm{cm}^{2}$ of coral branch surface area after quality control as described for proteins. 
Lipid analysis

Lipids were extracted using a modified version of Harland et al. (1993). Ten $\mathrm{ml}$ of coral

340 slurry was freeze dried overnight then homogenised by vortexing with $2 \times 5 \mathrm{~mL}$ of 2:1

341 dichloromethane:methanol (DCM:MeOH), each left for 24 hours at $4^{\circ} \mathrm{C}$. Extracts were filtered to

342 remove any debris using a glass filter (Whatman GF/C) and purified once with $5 \mathrm{ml} 0.88 \%$

$343 \mathrm{KCl}: \mathrm{H}_{2} \mathrm{O}$ and thrice with 1:1 MeOH: $\mathrm{H}_{2} \mathrm{O}$. Total lipid content was determined gravimetrically from

344 dried samples $\left(60^{\circ} \mathrm{C}\right.$ overnight $)$ in pre-weighed acetone washed aluminum trays. Total lipid

345 content was expressed in $\mathrm{mg}$ per $\mathrm{cm}^{2}$ of coral branch surface area.

MBD-seq library preparation

349 thiocyanate, $30 \mathrm{mM}$ sodium citrate, $30 \mathrm{mM} \beta$-mercaptoethanol) followed by phenol chloroform

350 purification and a final cleanup with Zymo Genomic DNA Clean and Concentrator-10 kit (Catalog

351 No D4011). Genomic DNA was sheared using a Misonix Sonicator 3000 to a size range of 200 to

$352800 \mathrm{bp}$ checked by gel electrophoresis. Enrichment reactions were performed using the

353 MethylCap kit (Diagenode Cat. No. C02020010) with an initial input of $2 \mu \mathrm{g}$ of sheared DNA per

354 reaction. The methylated fraction was eluted from the capture beads in a single step using High

355 Elution Buffer. Library preparation using NEBNext ${ }^{\circledR}$ DNA library Prep Master Mix Set (E6040L)

356 and sequencing on an Illumina HiSeq4000 was performed at the University of Texas Genome

357 Sequencing and Analysis Facility. Our total sample size was $\mathrm{N}=44$ (22 colonies divided in half 
358 giving 11 samples per treatment group). For the majority of these we sequenced only the

359 enriched library eluted from the capture beads. Fold coverages from these captured libraries

360 were used to estimate relative differences in GBM between samples. For a subset of 12 of the

36144 samples, we sequenced both the captured and flow-through fractions. Fold differences

362 between these captured and flow-through libraries were used to estimate absolute levels of

363 methylation across genes. We did this for only a subset of samples because we were primarily

364 interested in relative differences in GBM between groups. As relative differences could be

365 reasonably well assessed without sequencing the flow-through (Figure S2), we chose to focus

366 our sequencing resources on increasing sample size rather than more thorough estimates of

367 absolute methylation levels.

368

369 Repeated elements annotation

370 De novo identification of repeated elements in A. digitifera genome was performed using

371 RepeatModeler (http://www.repeatmasker.org/RepeatModeler/, version 1.0.10).

372 RepeatModeler launches two repeat finding programs, RECON 1.05 (28)

373 (http://eddylab.org/software/recon/) and RepeatScout 1.0.5 (29)

374 (https://bix.ucsd.edu/repeatscout/), to generate a genomic database and upon which to build,

375 refine and classify consensus models of putative interspersed repeats. This pipeline produced a

376 total of 2,858 non-redundant repeat families in recent $A$. digitifera genome sequence. These

377 sequences were then used as the custom library to search the $A$. digitifera genome using 
378 RepeatMasker (http://www.repeatmasker.org/). Approximate 7.33\% and 5.47\% of the genome

379 was annotated to be composed of retrotransposon and DNA transposon elements, respectively.

MBD-seq data processing

Forty four MBD-seq libraries were prepared from reciprocally transplanted coral

383 fragments. Sequencing produced 980 million raw reads with a mean of $16 \pm 0.65$ SEM million

384 reads per sample. Raw reads were trimmed of non-template sequence using Cutadapt (30) and

385 quality filtered using Fastx toolkit (http://cancan.cshl.edu/labmembers/gordon/fastx toolkit/).

386 Adapter trimming and quality filtering reduced these the total read count 940 million, mean =

$38715 \pm 0.64$ SEM million reads per sample. The reference genome and annotations (version 1.1)

388 for Acropora digitifera (31) were downloaded from NCBI. Trimmed and filtered reads were

389 mapped to this concatenated reference using Bowtie2 (32). To ensure that using a reference

390 from an alternate species did not severely impair mapping, we compared the mean mapping

391 efficiency against the $A$. digitifera reference with that of a draft genome sequence for $A$.

392 millepora produced by David Miller and coworkers (James Cook University). Mean mapping

393 efficiency against the $A$. digitifera $(78.4 \pm 0.7 \%$ SEM) reference was only $5.2 \%$ lower than that of

394 A. millepora ( $83.6 \pm 0.8 \%$ SEM), hence sequence differences between the two species did not

395 appear to substantially impair read mapping. Following mapping, PCR duplicates were removed

396 using Picard (https://broadinstitute.github.io/picard/). Mean duplication frequency was $14.3 \pm$

$3970.5 \%$ SEM. The BAM files were filtered to retain only highly uniquely aligned reads (mapping

398 quality 30 , or $0.1 \%$ chance of non-unique alignment) using samtools (33), after which the 
399 number of reads overlapping various genomic regions - exon, intron, genic (exon + intron),

400 repeated elements longer than 500b, and repeat-free intergenic regions at least $1 \mathrm{~kb}$ long and at

401 least $2 \mathrm{~kb}$ away from any gene - were counted using BEDtools (34).

402

403

MBD-score and GBM gene classes

404

For 12 samples (3 per each experimental group) we quantified absolute levels of GBM as

405 the $\log _{2}$ fold difference in coverage between captured and flow-through DNA fractions while

406 controlling for genotype, as described in Dixon et al. (2016). These values were used as gene-

407 specific "MBD scores" throughout the study. As expected based on previous studies $(3,35)$,

408 MBD-scores were bimodally distributed (Fig. S1 A) and negatively correlated with $\mathrm{CpG}_{\mathrm{o} / \mathrm{e}}$ (Figure

4091 D) providing genome-wide validation for our MBD-seq technique. The MBD-score threshold

410 separating the two clouds of high point density in the scatter plot on Figure $1 \mathrm{D}$ was used to

411 delineate the two gene classes for computation of the "GBM class difference" (GBMcd) for each

412 sample (Fig. 2 I, J).

413

414 Tag-seq data processing

415 Transcription was quantified using Tag-seq (Meyer et al. 2011; Lohman et al. 2016). The

416 Tag-seq reads were downloaded from the SRA database (accession SRP049522; (23)) and

417 mapped against the A.digitifera genome using SHRiMP (37). Mapped reads overlapping

418 annotated coding sequences were counted using intersection-nonempty method in HTseq 
419 version 0.6.1p1 (38). Normalization of raw counts and statistical analyses were performed using

420 DESeq2 (39).

421

422 Assessing variation in gene body methylation and transcription

423 As with gene expression analyses, normalization and statistical analyses of MBD-seq

424 reads were performed with DESeq2 (39). To test for origin effects, we performed two tests that

425 compared the two groups placed at Keppel to each other (OK vs KK) and the two groups placed

426 at Orpheus to each other (OO vs KO). These tests were intended to identify effects of origin

427 while controlling for environmental conditions experienced during the experiment. To assess

428 effects of transplantation, we compared groups that originated from Orpheus to each other (OO

429 vs OK) and groups that originated from Keppel to each other (KK vs KO). For these tests, we

430 included an additional parameter of colony identity to identify effects of transplantation while

431 controlling for genotype. For TagSeq, only genes with mean read count $\geq 3$ were considered for

432 analysis (19706 genes), and for MBD-seq we chose genes with mean read count $\geq 20$ (27084

433 genes).

434

435 Discriminant analysis of principal components

436 GBM data were further analyzed using discriminant analysis of principal components

437 (DAPC) implemented in the R package adegenet (40), following the procedures outlined in

438 Kenkel \& Matz (2016). DAPC is a multivariate analysis method designed to identify between-

439 group variation while neglecting within-group variation. We used this method to distill our 
440 multivariate MBD-seq dataset into single axes that maximized discrimination between natives

441 from the two experimental sites (KK and OO samples). The result was a discriminant axis

442 contrasting the GBM variation of the two native populations - with one pole designating native

443 Keppel-like GBM patterns and the opposite pole designating native Orpheus-like patterns. The

444 function was then applied to data from the transplanted samples, so that their positions along

445 discriminant axis described their similarity in GBM patterns to the contrasted native

446 populations. It seems plausible that, either due to natural selection or plasticity, native corals

447 would possess patterns optimal for their particular site. If this is true, then transplants with

448 DAPC values closer those of local corals would be expected to show improved fitness proxies

449 (eg: samples transplanted to Keppel with DAPC values closer to the 'Keppel-like' pole of the

450 discriminant axis would be expected to show higher fitness proxies and vice versa for samples

451 transplanted to Orpheus). 'DAPC Similarity' values describing each transplant's proximity along

452 the discriminate axis to natives of their transplantation site (Fig. 4 D) were computed by taking

453 the absolute value of the difference between the transplants' DAPC values and the mean value

454 for natives of their respective transplant sites, converting these distances into z-scores, and

455 multiplied them by -1 . The DAPC Similarity value for sample $X$ was:

456

457

$$
D_{A P C} \text { Similarity }_{x}=-1 \times\left(\left|D_{x}\right|-\bar{D}\right) / \sigma
$$

459 where, $D_{x}$ is its distance along the discriminant axis between sample $\mathrm{X}$ and the mean DAPC

460 value for natives of its transplantation site, $\bar{D}$ is the mean absolute distance for all transplants, 
461 and $\sigma$ is the standard deviation of absolute distance for all transplants. Correlations of these

462 values with fitness characteristics of transplanted fragments (Fig. $4 \mathrm{E}$ ) were computed using

463 function envfit ( $R$ package vegan (42))

464

465 Validation of MBD-seq results by bisulfite amplicon sequencing

466 To validate our MBD-seq results, we used targeted bisulfite sequencing. Genomic DNA

467 for this procedure was extracted and purified as described above. It should be noted that as

468 with the MBD-seq and Tag-seq, targeted bisulfite sequencing was performed from separate

469 isolations from the same or closely adjacent branch. We performed bisulfite conversion using an

470 EZ DNA Methylation-Gold kit (Zymo Research; cat. no. D5005). Thermocycler conditions for

471 conversion were $98^{\circ} \mathrm{C}$ for 10 minutes followed by $53^{\circ} \mathrm{C}$ for 4 hours. Primers for post-conversion

472 amplification were designed using Bisulfite Primer Seeker (Zymo Research;

473 http://www.zymoresearch.com/tools/bisulfite-primer-seeker). Primer sequences are given in

474 Table S1. We designed 13 primer sets to target coding sequences (excised from the $A$. digitifera

475 reference genome) of 13 separate loci. Primer sequences included 5' tails for downstream

476 addition of barcodes and Illumina adapters by PCR. Target loci were selected based on either

477 strong origin or transplant effects in the DESeq2 analyses. Amplification from bisulfite converted

478 samples was performed using EpiMark Hot Start Taq DNA Polymerase (New England Biolabs;

479 cat. no. M0490S). Thermocycler conditions were $95^{\circ} \mathrm{C}$ for 30 seconds followed by $35-40$ cycles

480 of $95^{\circ} \mathrm{C}$ for 15 seconds, $53^{\circ} \mathrm{C}$ for 30 seconds, $68^{\circ} \mathrm{C}$ for 30 seconds. Barcodes and adapters for

481 multiplexed sequencing were added to the resulting PCR products in a second PCR. The 
oligonucleotide sequences used for barcoding were the same as those given in the Tag-seq

483 library preparation (Meyer et al. 2011; https://github.com/z0on/tag-based_RNAseq).

484 Sequencing was performed using paired-end 600 cycle runs on the Illumina Miseq. Resulting

485 reads were quality trimmed using cutadapt (30). Quantification of CpG methylation was

486 performed using Bismark (43) using a fasta file of the 13 exon sequences used to design the

487 primers as a reference. For a given sample, CpG sites represented by fewer than 50 reads were

488 excluded from the analysis (2022 low coverage calls excluded). The difference in means based

489 on origin for all CpGs in each gene correlated with the difference in mean normalized counts

490 from the MBD-seq reads (Figure 1 D).

491

492 Primers used for targeted bisulfite sequencing. Each primer includes a 5' tail (bold) for

493 amplification with barcoded primers for multiplex Illumina sequencing included in the Tag-seq

494 library preparation.

\begin{tabular}{ll}
\hline Primer & Sequence (5'-3') \\
\hline LOC107327073_bsfTag_For & CTACACGACGCTCTTCCGATCTTTTTTGYGAGGTTGATTTTGTTATTATG \\
LOC107327073_bsfTag_Rev & ACGTGTGCTCTTCCGATTTTCCAAACATATTCCTTTCCATAACATTC \\
LOC107356898_bsfTag_For & CTACACGACGCTCTTCCGATCTTAGATTTYGTTATAATGTTATTAAGAAGTGAAGG \\
LOC107356898_bsfTag_Rev & ACGTGTGCTCTTCCGATAATAATATTAAACATTCTCTCTACAAATCTACCAC \\
LOC107358158_bsfTag_For & CTACACGACGCTCTTCCGATCTTGGTYGGATTGTTGAAGAGTTTAAGTAG \\
LOC107358158_bsfTag_Rev & ACGTGTGCTCTTCCGATACACCCAAATCACCCATCTCATTAAC \\
LOC107356899_bsfTag_For & CTACACGACGCTCTTCCGATCTGTTTTTAAAATTTGAAGGATTTGGTTTTGTTG \\
LOC107356899_bsfTag_Rev & ACGTGTGCTCTTCCGATTTACATTATATTTTCCAAACATATTTCATACCATAAC \\
LOC107358871_bsfTag_For & CTACACGACGCTCTTCCGATCTGATATYGGGTTTTTAATAATAATTGTATGTTGGTTG \\
LOC107358871_bsfTag_Rev & ACGTGTGCTCTTCCGATTTTTCCTTTAAAATTATTTCCACCCAACTCC \\
LOC107334334_bsfTag_For & CTACACGACGCTCTTCCGATCTATAAGGATATGTAGGGTTTTGGTAAGG \\
LOC107334334_bsfTag_Rev & ACGTGTGCTCTTCCGATAATTCATAATAACTACCCTACAACAAAAAATCCC \\
LOC107347512_bsTag_For & CTACACGACGCTCTTCCGATCTATYGATAGATTAAAAGAAGTTGGAGTATTG
\end{tabular}




$\begin{array}{ll}\text { LOC107347512_bsTag_Rev } & \text { ACGTGTGCTCTTCCGATCCTAAACAATCCATAAAAACCTTCCTACAATTC } \\ \text { LOC107350794_bsTag_For } & \text { CTACACGACGCTCTTCCGATCTGAYGTGTTTTGTATTTAGTTATTGGATATTTGG } \\ \text { LOC107350794_bsTag_Rev } & \text { ACGTGTGCTCTTCCGATATCTTCCRCAATTAAAAAAACATACTTAAAATCTTCAC } \\ \text { LOC107339795_bsTag_For } & \text { CTACACGACGCTCTTCCGATCTTGAATATAGTTAAGGTAAATGGATGAGTTATATATGG } \\ \text { LOC107339795_bsTag_Rev } & \text { ACGTGTGCTCTTCCGATCAAAAATAAACRAATCAAACAAAACAATCATTAC } \\ \text { LOC107336909_bsTag_For } & \text { CTACACGACGCTCTTCCGATCTAGAGATGYGGAATAGATATTTTTGGTTGG } \\ \text { LOC107336909_bsTag_Rev } & \text { ACGTGTGCTCTTCCGATAATAACRAACATTACATCTTATTTCTCTAAAATAATAC } \\ \text { LOC107352877_bsTag_For } & \text { CTACACGACGCTCTTCCGATCTTGGTGTYGTGAATTTGTTTAAATAATTTATG } \\ \text { LOC107352877_bsTag_Rev } & \text { ACGTGTGCTCTTCCGATCATTCCATCRACACAACTAAATAAAAAC } \\ \text { LOC107351808_bsTag_For } & \text { CTACACGACGCTCTTCCGATCTGTYGAGATATGTTAAAATTTGAGGATGTGAGTTTG } \\ \text { LOC107351808_bsTag_Rev } & \text { ACGTGTGCTCTTCCGATATCCACCATTCRCCAACTTACTAATAAATCTCCC } \\ \text { LOC107327285_bsTag_For } & \text { CTACACGACGCTCTTCCGATCTATTATGTTAGYGTTTTTGTTATTTTGGATTGTGG } \\ \text { LOC107327285_bsTag_Rev } & \text { ACGTGTGCTCTTCCGATCCTCATTCACAAAAACAACCTTTAATC }\end{array}$

496 Analysis of genetic differentiation

The MBD-seq reads were also used to analyze genetic distances among the 22

498 sequenced colonies. For this procedure, we concatenated reads from clone pairs into single

499 files, and mapped the reads to the A. digitifera reference genome (31) using Bowtie2 (32). For

500 the subset of 12 samples where we sequenced both captured and flow-through fractions, both

501 sets of reads were concatenated for genotyping. Between-individual genetic distances for DAPC

502 analysis were determined as (1 - Identity-By-State) using ANGSD (44) with the following filter

503 settings: -uniqueOnly 1 -remove_bads 1 -minMapQ 20 -minQ 30 -baq 1 -minlnd 10 -snp_pval 1e-

5042 -minMaf 0.1 (to summarize, only high-quality and uniquely-mapped reads were taken into

505 account; only sites covered in a minimum of 10 individuals were considered; the cutoff for the

$506 \mathrm{p}$-value for the SNP being true was set at 0.01; and only alleles with frequency equal or

507 exceeding 0.1 were used). This resulted in genotyping information for roughly 507,000

508 polymorphic sites. The between-population $F_{\mathrm{ST}}$ for genes passing $10 \%$ FDR cutoff for either for 
509 origin or transplant effect and a random subset of 500 non-significant genes (rather than whole

510 genome, to speed up computation) were determined using ANGSD based on site frequency

511 spectra as priors, which were generated for every gene subset without using filters that would

512 affect representation of alleles of different frequencies (-uniqueOnly 1 -remove_bads 1 -

513 minMapQ 20 -minQ 30 -baq 1 -minlnd 4).

514

515 Determination of relative proportions of Symbiodinium clades

516 The MBD-seq reads were mapped to the concatenated reference including A. digitifera

517 genome and four Symbiodinium sp. trasncriptomes from four different genotypic groups

518 ("clades"). Transcriptomes for Symbiodinium clades A and B were from (45) and transcriptomes

519 for clades C and D were from (46). We then counted the relative proportions of reads producing

520 highly unique matches (mapping quality 40 or higher) to each Symbiodinium transcriptome,

521 using a custom perl script zooxType.pl. All corals were found to be dominated by Symbiodinium

522 clade C.

523

524 Data and scripts availability

525 TagSeq, MBD-seq, and amplicon-bisulfite sequencing datasets are available through NCBI

526 Short Read Archive (Project Accession: SRP049522). Scripts and traits data are available in a

527 GitHub repository dedicated to this paper

528 (https://github.com/grovesdixon/reciprocal_transplant_methylation). 
530 References (Materials and Methods)

531 23. G. B. Dixon, L. K. Bay, M. V Matz, Bimodal signatures of germline methylation are linked

532 with gene expression plasticity in the coral Acropora millepora. BMC Genomics. 15, 1109

533 (2014).

534 24. P. Jokiel, J. Maragos, L. Franzisket, in Coral reefs: research methods, D. R. Stoddart, R. E. 535 Johannes, Eds. (UNESCO, Paris, ed. 5, 1978), pp. 529-542.

$53625 . \quad J$. Stimson, R. A. Kinzie, The temporal pattern and rate of release of zooxanthellae from 537 the reef coral Pocillopora damicornis (Linnaeus) under nitrogen-enrichment and control conditions. J. Exp. Mar. Bio. Ecol. 153, 63-74 (1991).

539 26. T. Masuko et al., Carbohydrate analysis by a phenol-sulfuric acid method in microplate format. Anal. Biochem. 339, 69-72 (2005).

$54127 . \quad$ A. D. Harland, J. C. Navarro, P. S. Davies, L. M. Fixter, Lipids of some Caribbean and Red Sea corals: total lipid, wax esters, triglycerides and fatty acids. Mar. Biol. 117, 113-117 (1993).

28. Z. Bao, S. R. Eddy, Automated de novo identification of repeat sequence families in sequenced genomes. Genome Res. 13, 1269-1276 (2003).

29. A. L. Price, N. C. Jones, P. A. Pevzner, De novo identification of repeat families in large genomes. Bioinformatics. 21 (2005), doi:10.1093/bioinformatics/bti1018.

30. M. Martin, Cutadapt removes adapter sequences from high-throughput sequencing reads. EMBnetjournal. 17, 10-12 (2011).

31. C. Shinzato et al., Using the Acropora digitifera genome to understand coral responses to environmental change. Nature. 476, 320-3 (2011).

32. B. Langmead, S. L. Salzberg, Fast gapped-read alignment with Bowtie 2. Nat. Methods. 9, 357-359 (2012).

554 33. H. Li et al., The Sequence Alignment/Map format and SAMtools. Bioinformatics. 25, 2078-9 (2009).

556 34. A. R. Quinlan, I. M. Hall, BEDTools: A flexible suite of utilities for comparing genomic features. Bioinformatics. 26, 841-842 (2010). 
$558 \quad 35 . \quad$ G. B. Dixon, L. K. Bay, M. V. Matz, Evolutionary Consequences of DNA Methylation in a Basal Metazoan. Mol. Biol. Evol. 33 (2016), doi:10.1093/molbev/msw100.

560

36. B. K. Lohman, J. N. Weber, D. I. Bolnick, Evaluation of TagSeq, a reliable low-cost alternative for RNAseq. Mol. Ecol. Resour. 16, 1315-1321 (2016).

37. S. M. Rumble et al., SHRiMP: Accurate mapping of short color-space reads. PLoS Comput. Biol. 5, 1-11 (2009).

38. S. Anders, P. T. Pyl, W. Huber, HTSeq-A Python framework to work with high-throughput sequencing data. Bioinformatics. 31, 166-169 (2015).

39. M. Love, W. Huber, S. Anders, Moderated estimation of fold change and dispersion for RNA-Seq data with DESeq2. Genome Biol. (2014) (available at http://www.biomedcentral.com/content/pdf/s13059-014-0550-8.pdf).

40. T. Jombart, S. Devillard, F. Balloux, Discriminant analysis of principal components: a new

41. C. Kenkel, M. V Matz, Enhanced gene expression plasticity as a mechanism of adaptation to a variable environment in a reef-building coral. Nat. Ecol. Evol. 1, 0014 (2016).

42. J. Oksanen et al., vegan: Community Ecology Package. R Packag. ver. 2.4-3 (2017), , doi:10.4135/9781412971874.n145.

43. F. Krueger, S. R. Andrews, Bismark: A flexible aligner and methylation caller for BisulfiteSeq applications. Bioinformatics. 27, 1571-1572 (2011).

44. T. Korneliussen, A. Albrechtsen, R. Nielsen, ANGSD: Analysis of Next Generation Sequencing Data. BMC Bioinformatics. 15, 356 (2014).

45. T. Bayer et al., Symbiodinium transcriptomes: genome insights into the dinoflagellate symbionts of reef-building corals. PLoS One. 7, e35269 (2012).

46. J. T. Ladner, D. J. Barshis, S. R. Palumbi, Protein evolution in two co-occurring types of Symbiodinium: an exploration into the genetic basis of thermal tolerance in Symbiodinium clade D. BMC Evol. Biol. 12, 217 (2012). (2008), doi:10.1371/journal.pgen.1000144. 
Table S1. Sample sizes

\begin{tabular}{|c|c|c|c|}
\hline Sample group & N (TagSeq) & $\begin{array}{c}\text { N (MBD-seq, } \\
\text { captured and flow- } \\
\text { through fractions) }\end{array}$ & $\begin{array}{c}\text { N (MBD-seq, only } \\
\text { captured fraction) }\end{array}$ \\
\hline KK & 14 & 3 & 8 \\
\hline KO & 15 & 3 & 8 \\
\hline OK & 15 & 3 & 8 \\
\hline OO & 15 & 3 & 8 \\
\hline
\end{tabular}

588

A

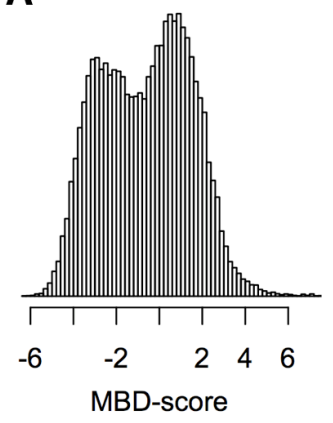

B

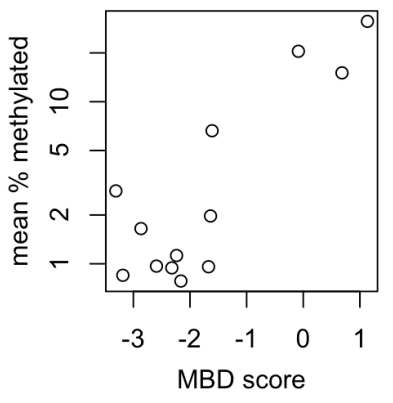

C

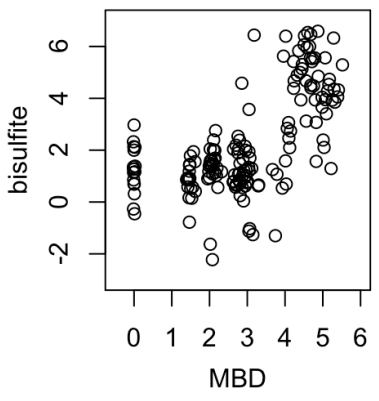

591 Figure S1. Distribution of MBD-scores $(A)$ and validation by bisulfite sequencing of amplicons (B,

592 C). B: Mean percent methylation was calculated as the proportion of methylated CpG sites

593 within each gene averaged across all samples. C: Correlation between MBD-based and bisulfite-

594 based measurement of the effects of each coral individual on methylation for each of the

595 assayed genes. 

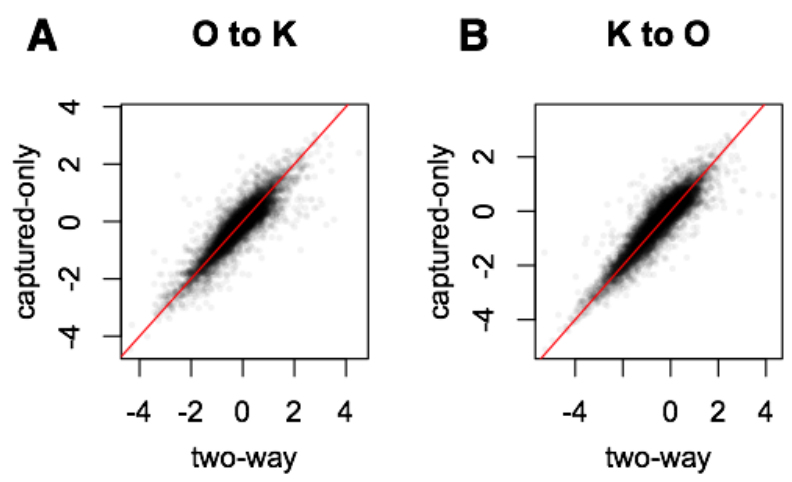

597 Figure S2 Correlation between inferred changes in GBM based on comparison of MBD-captured

598 and flow-through libraries ( $x$-axis) and only on MBD-captured fraction ( $y$-axis). The red line is 1:1

599 correspondence. (A) Orpheus to Keppel transplantation; (B) Keppel to Orpheus transplantation.

600 These plots were generated for a subset of 12 samples (three for each of the four experimental

601 groups, KK, KO, OK, and OO) for which both the captured and the flow-through fractions were

602 sequenced. 


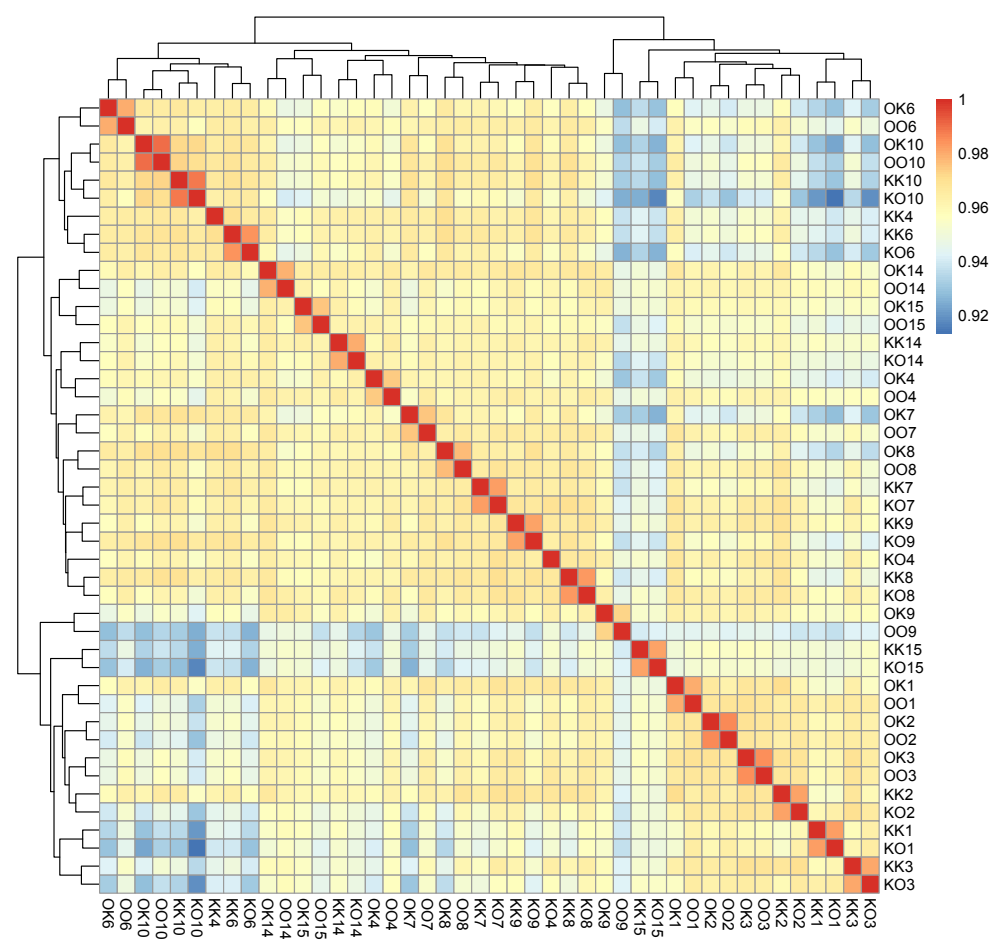

604 Figure S3 Heatmap of GBM correlations among samples, illustrating strong dependence of GBM on genotype. Colors indicate Spearman's rank correlations for normalized MBD-seq read counts

606 across all coding genes $(\mathrm{N}=24853)$; the lowest observed correlation value was 0.9. Samples

607 were clustered by maximum distance method. First letter of sample names indicates sample

608 origin, second letter indicates transplantation site, and number indicates replicate. Samples

609 sharing the same first letter and the same number are clonal fragments from the same colony.

610 All of the 22 clone pairs except one (sample pair KK4 and KO4) clustered together. 


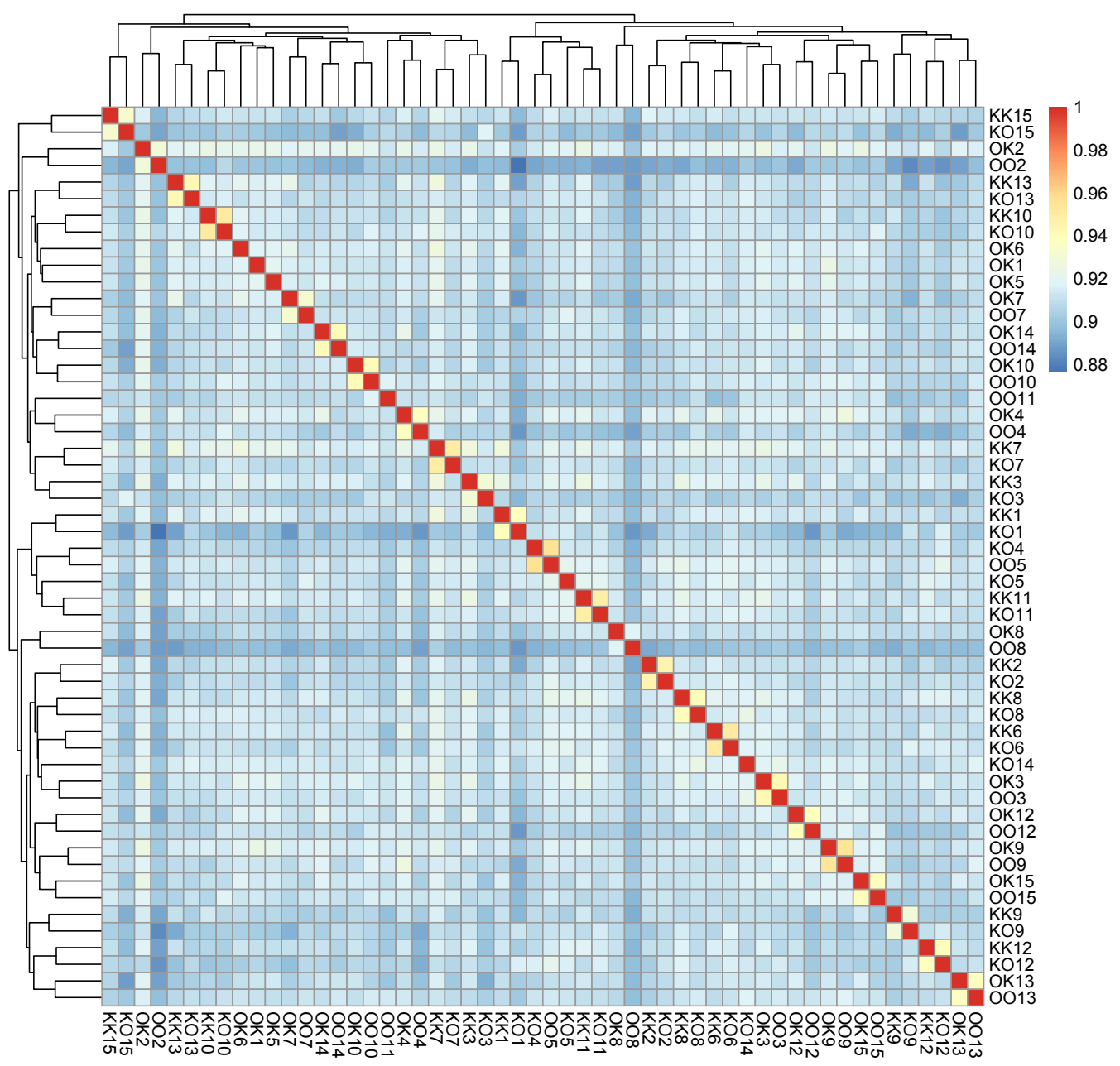

613 Figure S4 Heatmap of overall correlations in transcription illustrating strong dependence of

614 transcription on genotype. Colors indicate Spearman's correlations for normalized Tag-seq read

615 counts across all coding genes $(\mathrm{N}=19706)$. Samples were clustered by maximum distance

616 method. First letter of sample names indicates sample origin, second letter indicates

617 transplantation site, and number indicates replicate. Samples sharing the same first letter and

618 number are clonal fragments from the same colony. All 24 available clone pairs clustered

619 together (six samples lacked data for clone pairs). 

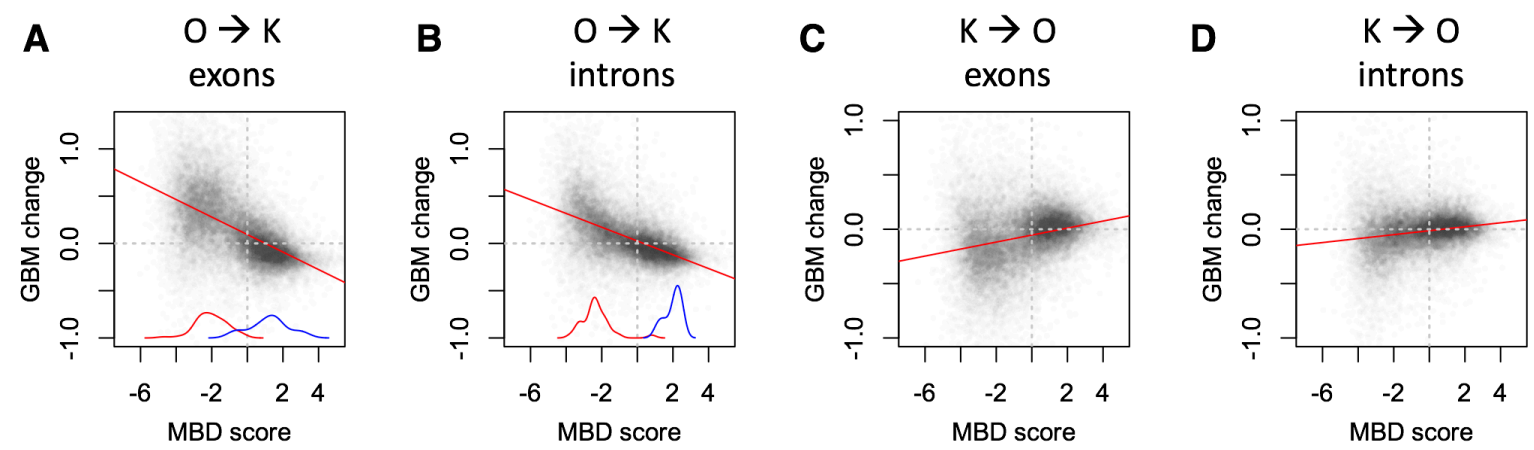

623 Figure S5. GBM $\log _{2}$ fold-changes change in response to transplantation in exons and introns

624 plotted against MBD-score for each gene. Red and blue curves near the $\mathrm{x}$-axis are density plots 625 of significantly up- and down-regulated genes.

626

A $\quad O \rightarrow K$

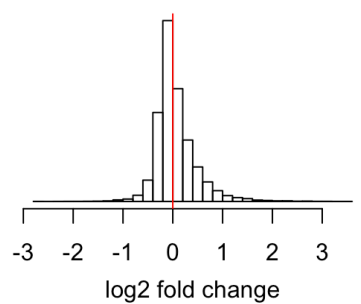

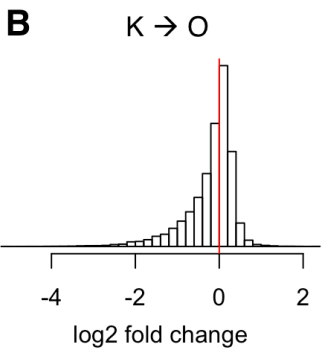

C

$\mathrm{O} \rightarrow \mathrm{K}$

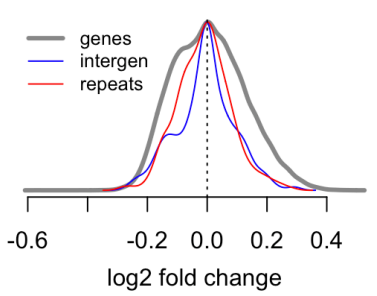

D $\quad K \rightarrow O$

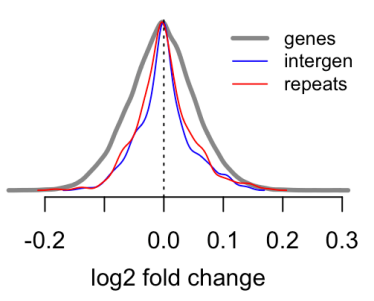

628 Figure S6. Absolute methylation changes in response to transplantation ("away" vs. "home")

629 can be positive or negative across genes (A, B) and affect mostly protein-coding regions (C, D).

630 Plots on $A$ and $B$ are based on comparison between captured and flow-through fractions for 12

631 samples (three for each of the four experimental groups, KK, KO, OK, and OO). Plots on panels C

632 and D were generated based on captured-only analysis of all 44 MBD-seq samples (Table S1). 
A $\quad \mathrm{O} \rightarrow \mathrm{K}$

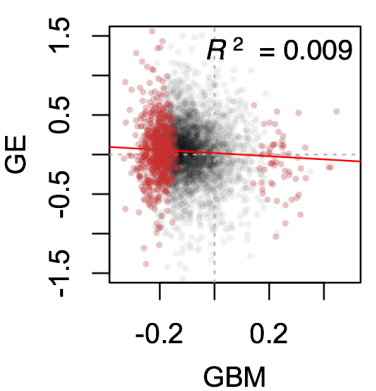

B $\quad K \rightarrow O$

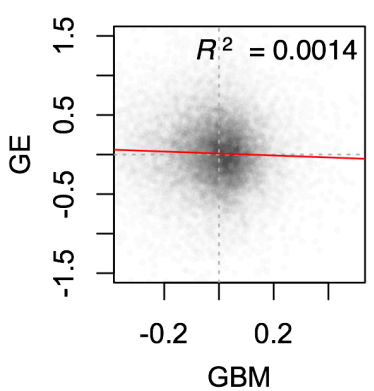

635 Figure S7. Gene expression changes in response to transplantation are negatively correlated

636 with GBM changes. Both correlations are highly significant $(p<0.0001)$. Red points on panel A

637 represent genes with statistically significant (FDR<10\%) change in GBM. 
A

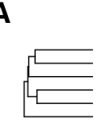

Constitutive ( [KK,KO] vs. [OO,OK] ): GE

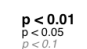

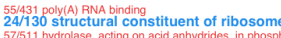

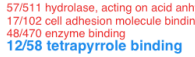

B

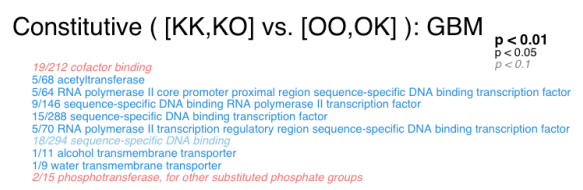

C

Plastic (OK vs. OO): GE
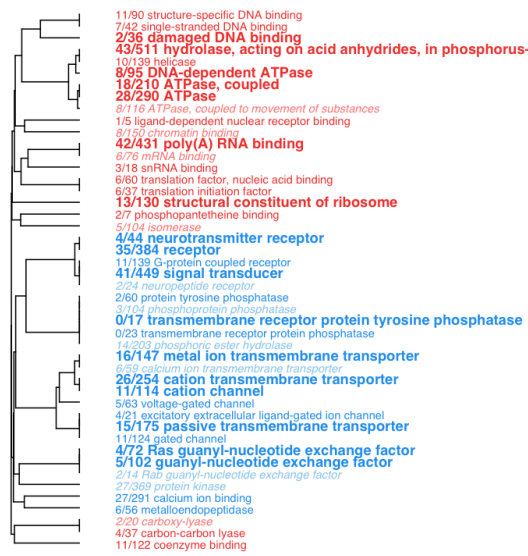

D Plastic (OK vs OO): GBM

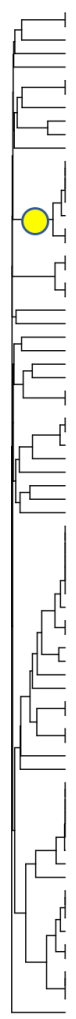

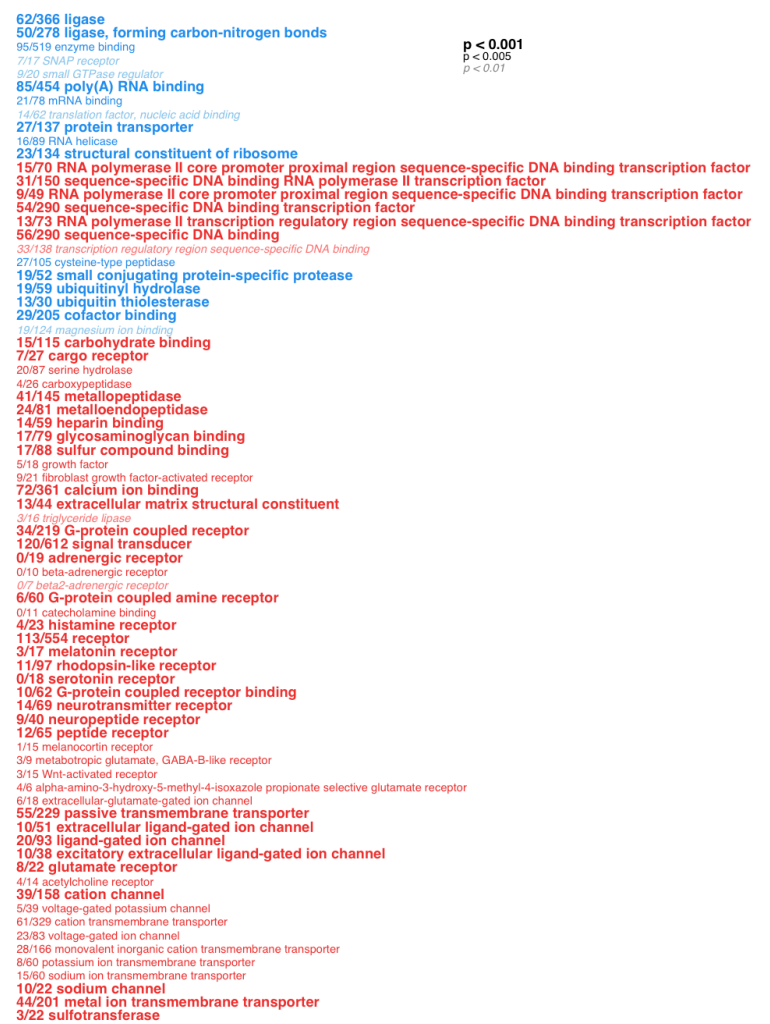

640 Figure S8. Gene ontology categories significantly enriched with up- or down-regulated genes

641 (red and blue, respectively) in GE and GBM comparisons. The Benjamini-Hochberg corrected

642 significance of enrichment is indicated by the size and type of the font (see legends). Note that

643 for plastic GBM the significance thresholds are ten times more stringent, to fit the GO plot onto

644 one page. The fraction preceding the category name is the number of genes exceeding raw $\mathrm{p}$ -

645 value of 0.05 relative to the total number of genes in the category. The dendrograms are

646 hierarchical clustering of GO categories based on proportion of shared genes (47). Genes

647 comprising the branch labelled by the yellow circle (transcription factors) show plastic changes

648 (D) that are opposite to the constitutive differences between populations (B). 


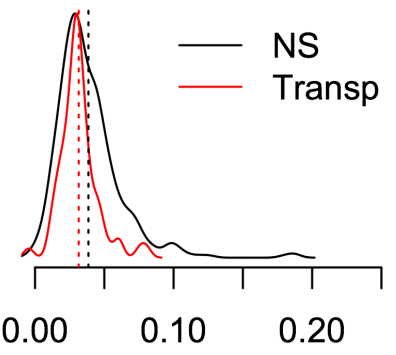

651 Figure S9. Density plots of $F_{\mathrm{ST}}$ for genes that show significant (FDR $<0.1$ ) GBM change in

652 response to transplantation from Orpheus to Keppel (“Transp", red curve) compared to 500

653 randomly picked non-significant genes ("NS", black curve). Dashed vertical lines mark the mean 654 of each dataset.

655 